\title{
Measurement of body fat using leg to leg bioimpedance
}

\author{
R Y T Sung, P Lau, C W Yu, P K W Lam, E A S Nelson
}

\begin{abstract}
Aims-(1) To validate a leg to leg bioimpedance analysis (BIA) device in the measurement of body composition in children by assessment of its agreement with dual energy $x$ ray absorptiometry (DXA) and its repeatability. (2) To establish a reference range of percentage body fat in Hong Kong Chinese children. Methods-Sequential BIA and DXA methods were used to determine body composition in 49 children aged 7-18 years; agreement between the two methods was calculated. Repeatability for the BIA method was established from duplicate measurements. Body composition was then determined by BIA in 1139 girls and 1243 boys aged 7-16 years, who were randomly sampled in eight local primary and secondary schools to establish reference ranges.

Results-The 95\% limits of agreement between BIA and DXA methods were considered acceptable $(-3.3 \mathrm{~kg}$ to $-0.5 \mathrm{~kg}$ fat mass and -3.9 to $0.6 \%$ body fat). The percentage body fat increased with increasing age. Compared to the 1993 Hong Kong growth survey, these children had higher body mass index. Mean (SD) percentage body fat at 7 years of age was $17.2 \%(4.4 \%)$ and $14.0 \%(3.4 \%)$ respectively for boys and girls, which increased to $19.3 \%(4.8 \%)$ and $27.8 \%(6.3 \%)$ at age 16 .
\end{abstract}

Conclusion-Leg to leg BIA is a valid alternative method to DXA for the measurement of body fat. Provisional reference ranges for percentage body fat for Hong Kong Chinese children aged 7-16 years are provided.

(Arch Dis Child 2001;85:263-267)

Keywords: bioimpedance; body mass index; body fat

R Y T Sung

$\mathrm{C}$ W Yu

E A S Nelson

Department of Sports Science and Physical Education, The Chinese University of Hong Kong

P Lau

Centre for Clinical Trials and Epidemiological

Research, The Chinese

University of Hong

Kong

P K W Lam

Correspondence to: Dr Sung

yntzsung@cuhk.edu.hk estimation of body composition is relatively simple and less time consuming. BIA is based on the principle that the electrical conductivity through body fluid is much greater in fat free mass than in fat mass, because fat free mass contains all body fluids and electrolytes. Conventional BIA systems require four gel electrodes placed at upper and lower limbs, and the subject has to lie supine for the measurement. Newer leg to leg BIA devices measure impedance across the lower limbs, and the system's four electrodes are in the form of stainless steel foot pads mounted on the top surface of a platform scale. The subject only needs to stand barefoot on the scale for simultaneous measurement of body weight and impedance; with manual entry of the subject's gender and height into the system via a digital keyboard, the subject's percentage body fat is displayed immediately. Because of its convenience, this leg to leg BIA method of estimating body fat has become increasingly popular with health professionals, as well as the general public, for the assessment and monitoring of obesity. Nevertheless, there are few data on its agreement with other standard methods such as dual energy $x$ ray absorptiometry (DXA) in children, and normal reference values for percentage body fat in children estimated by BIA are not available.

The aims of the study were: (1) to validate a leg to leg BIA device in the measurement of body composition in children by assessment of its agreement with DXA, and its repeatability; and (2) to establish a reference range of percentage body fat derived from this BIA method in Hong Kong Chinese children.

\section{Methods}

SUBJECTS

For the validation of the BIA method in comparison with DXA, 49 children (17 obese, 32 non-obese) were recruited from the obesity clinic of the Prince of Wales Hospital and nearby schools. All children and their parents gave informed written consent prior to participation. After validation, 2382 healthy children from eight local schools, aged 7-16 years, were randomly sampled for BIA. One or two classes were randomly chosen from each school and BIA of all students in the chosen classes were measured. The study protocol was explained to the students by their physical education teachers, and measurements were taken after agreement by the students.

\section{PROTOCOL}

In the validity study, all measurements were performed by a single investigator (YCW). Sequential height, BIA, and DXA measurements were undertaken at least two hours after 
Table 1 Body composition analysis by DXA and BIA $(n=49)$

\begin{tabular}{lrrrrc}
\hline & Mean & \multicolumn{1}{c}{$S D$} & Median & Minimum & Maximum \\
\hline Age (y) & 13.0 & 3.1 & 13.8 & 5.9 & 17.3 \\
Height (cm) & 154.6 & 16.4 & 156.5 & 114.0 & 178.0 \\
Weight (kg) & & & & & \\
$\quad$ DXA & 60.9 & 26.5 & 53.3 & 19.8 & 120.1 \\
$\quad$ BIA & 60.2 & 26.2 & 52.5 & 19.6 & 118.6 \\
Fat mass (kg) & & & & & \\
$\quad$ DXA & 17.9 & 13.1 & 13.0 & 3.4 & 48.0 \\
$\quad$ BIA & 19.8 & 15.7 & 14.4 & 2.8 & 56.5 \\
FFM (kg) & & & & & \\
$\quad$ DXA & 41.4 & 15.1 & 41.7 & 15.7 & 71.1 \\
$\quad$ BIA & 40.4 & 13.4 & 39.7 & 16.8 & 74.8 \\
\% fat & 27.3 & 10.3 & 25.8 & 8.5 & 45.4 \\
$\quad$ DXA & 28.9 & 12.8 & 25.0 & 12.0 & 59.0 \\
$\quad$ BIA & & & & & \\
\hline
\end{tabular}

FFM, fat free mass (lean mass plus bone mineral content).

breakfast. Children were measured in light sport wear after they had emptied their bladders. Height was measured to the nearest 1 $\mathrm{mm}$ using a wall mounted stadiometer. BIA was performed with a Tanita BIA body fat analyser which incorporates weighing scales and measures both weight and bioimpedance (TBF-401, Tanita Co., Tokyo, Japan). Subjects were asked to stand barefoot on the metal sole plates of the machine, and gender and height details were entered manually into the system via a keyboard. Body weight and percentage body fat, estimated using the standard built in prediction equations for children, were displayed on the machine and printed out. Two consecutive measurements were made in 58 subjects to calculate the repeatability. DXA measurements were taken immediately after the BIA measurements on the same day. The DXA instrument was a Hologic QDR-4500 (Waltham, Massachusetts, USA). It gives readings of fat mass, lean mass, bone mineral content, total body mass (sum of fat mass, lean mass, and bone mineral content), and percentage body fat. The whole body scan time was three to four minutes using the fan beam model.

To obtain reference values for percentage body fat of Hong Kong children, the same BIA measurements were carried out in eight local primary and secondary schools using the same Tanita instrument.

Table 2 Repeatability of body composition measurements by BIA $(n=58)$

\begin{tabular}{lllll}
\hline & $\begin{array}{l}\text { Absolute mean } \\
\text { difference }\end{array}$ & $\begin{array}{l}\text { Within } \\
\text { subject SD }\end{array}$ & $\begin{array}{l}\text { p value for testing zero } \\
\text { mean difference }\end{array}$ & Repeatability ${ }^{*}(95 \%$ CI) \\
\hline Weight $(\mathrm{kg})$ & 0.01 & 0.07 & 0.59 & $0.19(0.16$ to 0.23$)$ \\
Fat mass $(\mathrm{kg})$ & 0.15 & 0.25 & 0.11 & $0.68(0.58$ to 0.84$)$ \\
FFM (kg) & 0.05 & 0.24 & 0.26 & $0.67(0.56$ to 0.81$)$ \\
$\%$ fat & 0.06 & 0.50 & 0.19 & $1.38(1.17$ to 1.69$)$ \\
\hline
\end{tabular}

^Repeatability describes, in $95 \%$ of the time, the maximum possible difference between any two measurements for the same subject.

Table 3 Agreement in body composition analysis between DXA and BIA $(n=49)$

\begin{tabular}{llll}
\hline & Mean difference & 95\% limits of agreement & $\begin{array}{l}\text { p value for testing no } \\
\text { differences }\end{array}$ \\
\hline Weight $(\mathrm{kg})$ & 0.71 & $(0.56$ to 0.86$)$ & $<0.0005$ \\
Fat mass $(\mathrm{kg})$ & -1.93 & $(-3.34$ to -0.52$)$ & 0.01 \\
FFM $(\mathrm{kg})$ & 0.99 & $(-0.39$ to 2.36$)$ & 0.16 \\
$\%$ fat & -1.65 & $(-3.92$ to 0.61$)$ & 0.15
\end{tabular}

* The differences were obtained by subtracting the data values measured by BIA from those measured by DXA.
STATISTICS

Repeatability (which may be referred to as "precision") for BIA measurements was estimated by the repeatability coefficient. ${ }^{1}$ Within subject variance $s$ was estimated from the one way analysis of variance table with subjects taken as a factor. ${ }^{2}$ The repeatability coefficient was obtained from the formula $2.77 \mathrm{~s}$. This describes, for $95 \%$ of the time, the maximum possible difference between any two repeated measurements for the same subject.

Agreement between BIA and DXA measurements was assessed by the limits of agreement. ${ }^{1}$ The mean difference between the two methods was known as the bias and $95 \%$ limits of agreement were calculated as the bias $\pm 2 \mathrm{SD}$ of the differences between methods.

The LMS method ${ }^{2}$ was used to construct centile curves for BMI and body fat. Smoothed values are determined by the $L, M$, and $S$ curves. The $\mathrm{L}$ curve determines the power of Box-Cox transformation required to normalize the distribution of body measurements at all ages. The $\mathrm{M}$ curve corresponds to the median values, while the $S$ curve shows the coefficient of variation. The smoothed centile curves are then computed using the normal score (for example, $z=1.282$ for the 75 th centile curve) and the information from the three curves.

The body mass index centiles of these 2382 children were then compared to those from a previous Hong Kong study, ${ }^{3}$ and to proposed international $\mathrm{BMI}$ standards derived from combined data from six countries, including the Hong Kong data. ${ }^{4}$ These proposed international BMI standards classified children as overweight or obese according to the cut off points defined to pass through BMI values of 25 and $30 \mathrm{~kg} / \mathrm{m}^{2}$ at age 18 .

These same international BMI cut off values for overweight and obesity, were then used to derive percentage of body fat cut off values for overweight and obesity as measured by BIA. Percentage body fat cut off values for overweight were also derived from the Hong Kong data alone using BMI cut off values of $25 \mathrm{~kg} / \mathrm{m}^{2}$ at age 18 and $23 \mathrm{~kg} / \mathrm{m}^{2}$ at age 18 . The latter lower cut off values have recently been suggested as being more appropriate to define overweight and obesity in Asian adults. ${ }^{5}$

\section{Results}

VALIDATION OF LEG TO LEG BIA

The BIA measurement of body composition was simple and well accepted by the children. Table 1 presents body composition characteristics of the 49 subjects. The repeatability of BIA, shown in table 2 , was $0.68 \mathrm{~kg}$ for fat mass and $1.4 \%$ for percentage body fat. Table 3 shows the agreement in body composition analysis between DXA and BIA. BIA significantly overestimated fat mass (difference obtained by subtracting data values measured by BIA from those by DXA: $-1.93 \mathrm{~kg}$ for a mean fat mass of $18.8 \mathrm{~kg}, 95 \%$ confidence interval (CI) -3.34 to $-0.52, \mathrm{p}<0.01)$. However, the bias of percentage body fat $(-1.65 \%)$ did not reach statistical significance. 
Table 4 Age distribution of 1243 boys and 1139 girls

\begin{tabular}{llrr}
\hline & \multicolumn{2}{c}{ Gender } & \\
\cline { 2 - 3 } Age & Boys & Girls & Total \\
\hline 7 & 115 & 114 & 229 \\
8 & 134 & 81 & 215 \\
9 & 124 & 129 & 253 \\
10 & 171 & 153 & 324 \\
11 & 116 & 111 & 227 \\
12 & 146 & 116 & 262 \\
13 & 144 & 121 & 265 \\
14 & 125 & 131 & 256 \\
15 & 104 & 105 & 209 \\
16 & 64 & 78 & 142 \\
Total & 1243 & 1139 & 2382 \\
\hline
\end{tabular}

REFERENCE RANGES FOR BMI AND PERCENTAGE BODY FAT MEASURED BY BIA

References ranges were generated from data on 1243 boys and 1139 girls aged 7-16 years (table 4). Figure 1 compares BMI centiles for boys and girls in the present sample with those from the 1993 Hong Kong growth study. It appears that the proportion of overweight children in Hong Kong has increased, particularly between the ages of 8 to 14 , with a more pronounced effect for boys than girls - that is, an upward bulge was noted in the higher
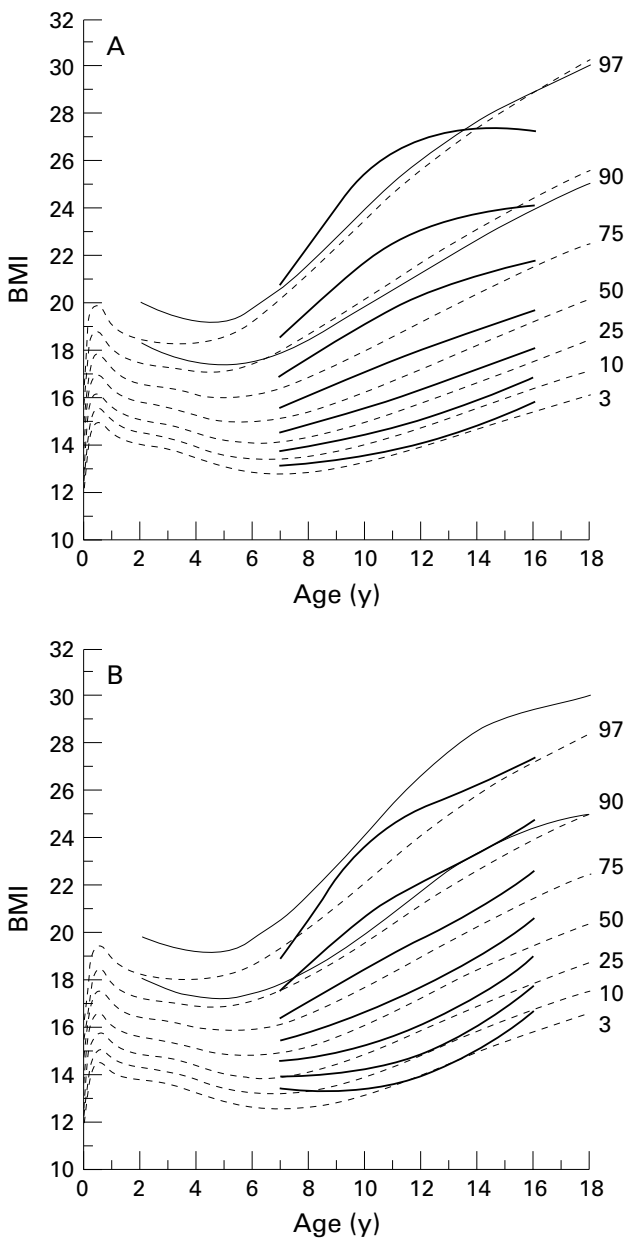

Figure 1 Comparison of BMI in 1243 boys $(A)$ and 1139 girls (B) with Hong Kong growth survey in 1993. Thick solid line, centile lines for present study; dotted line, centile lines for Hong Kong growth survey"; solid line, proposed international cut off values for overweight (extrapolated from BMI of $25 \mathrm{~kg} / \mathrm{m}^{2}$ at age 18) and obesity (extrapolated from BMI of $30 \mathrm{~kg} / \mathrm{m}^{2}$ at age 18). ${ }^{4}$
Table $5 L, M$, and $S$ values for the percentage body fat of 1243 boys and 1139 girls

\begin{tabular}{lllllllll}
\hline & \multicolumn{3}{l}{$L$} & \multicolumn{3}{l}{$M$} & & \multicolumn{2}{l}{$S$} \\
Age $(y)$ & Boys & Girls & & Boys & Girls & & Boys & Girls \\
\hline 7 & -0.20 & -0.28 & & 16.67 & 13.53 & & 0.25 & 0.24 \\
8 & -0.21 & -0.22 & & 16.50 & 14.67 & & 0.27 & 0.28 \\
9 & -0.21 & -0.16 & & 17.69 & 15.70 & & 0.29 & 0.31 \\
10 & -0.22 & -0.09 & & 19.25 & 16.74 & & 0.32 & 0.33 \\
11 & -0.24 & -0.02 & & 18.96 & 17.97 & & 0.34 & 0.33 \\
12 & -0.29 & 0.02 & & 17.60 & 19.39 & & 0.36 & 0.32 \\
13 & -0.36 & 0.04 & & 16.83 & 20.95 & & 0.35 & 0.30 \\
14 & -0.44 & 0.04 & & 15.99 & 22.69 & & 0.33 & 0.28 \\
15 & -0.53 & 0.02 & & 16.14 & 24.59 & & 0.29 & 0.26 \\
16 & -0.61 & 0.01 & & 18.37 & 26.56 & & 0.24 & 0.23 \\
\hline
\end{tabular}

centile curves. Table 5 lists the L, M, and S values of the 2382 children. Overweight was classified using three different cut off values: (1) proposed international BMI cut off value extrapolated from BMI $25 \mathrm{~kg} / \mathrm{m}^{2}$ at age 18 using data from six countries (BMI-25INT); (2) extrapolated from BMI $25 \mathrm{~kg} / \mathrm{m}^{2}$ at age 18 using only the 1993 Hong Kong growth survey data (BMI-25HK); and (3) extrapolated from BMI $23 \mathrm{~kg} / \mathrm{m}^{2}$ at age 18 using the 1993 Hong Kong growth survey data (BMI-23HK). The respective percentage of children in the present sample classified as overweight were $18 \%$ of boys and $12 \%$ of girls (BMI-25INT), $18 \%$ of boys and $15 \%$ of girls (BMI-25HK), and $30 \%$ of boys and $27 \%$ of girls (BMI-23HK). The highest rates of obesity were noted in boys aged $10-13$ years and girls aged $10-12$ years.

Percentage body fat in boys peaked at age 11 but in girls showed a steady increase from 7 to 16 years (fig 2). Table 6 shows the corresponding figures of percentage body fat for overweight or obese children as defined by the three different BMI cut off values.

Table 6 Cut off points for overweight given as percentage body fat measured with leg to leg BIA device

\begin{tabular}{llll}
\hline Age & $\begin{array}{l}\text { \% body fat at } \\
\text { BMI-25INT }\end{array}$ & $\begin{array}{l}\text { \% body fat at } \\
\text { BMI-25HKt }\end{array}$ & $\begin{array}{l}\text { \% body fat at } \\
\text { BMI-23HK }\end{array}$ \\
\hline Females & & & \\
7 & 20.5 & 20.5 & 15.8 \\
8 & 19.8 & 19.4 & 17.1 \\
9 & 21.5 & 21.5 & 18.5 \\
10 & 22.5 & 22.5 & 21.4 \\
11 & 23 & 23 & 21.5 \\
12 & 26.5 & 26.5 & 23.5 \\
13 & 28.5 & 27.5 & 23.5 \\
14 & 29.5 & 29.5 & 26.5 \\
15 & 33.5 & 32.5 & 29.5 \\
16 & 35.5 & 32.5 & 30.5 \\
Males & & & \\
7 & 20 & 20 & 20 \\
8 & 17.6 & 17.6 & 17.2 \\
9 & 22.7 & 22.7 & 19.9 \\
10 & 22.5 & 22.5 & 20.6 \\
11 & 22.5 & 22.5 & 22.5 \\
12 & 23 & 23 & 20.5 \\
13 & 21.5 & 21.5 & 20.5 \\
14 & 19.5 & 19.5 & 17.5 \\
15 & 25.5 & 25.5 & 17.5 \\
16 & 21.5 & 21.5 & 18.5 \\
\hline
\end{tabular}

$\star$ Derived from proposed international BMI cut off values defining overweight (extrapolated from $\mathrm{BMI}>25$ at age 18 years) based on data from six countries (BMI-25INT).

†Derived from BMI cut off values defining overweight (extrapolated from BMI $>25$ at age 18 years) based on Hong Kong data (BMI-25HK). ${ }^{3}$

‡Derived from BMI cut off values defining overweight (extrapolated from BMI $>23$ at age 18 years) based on Hong Kong data (BMI-23HK). ${ }^{3}$ 

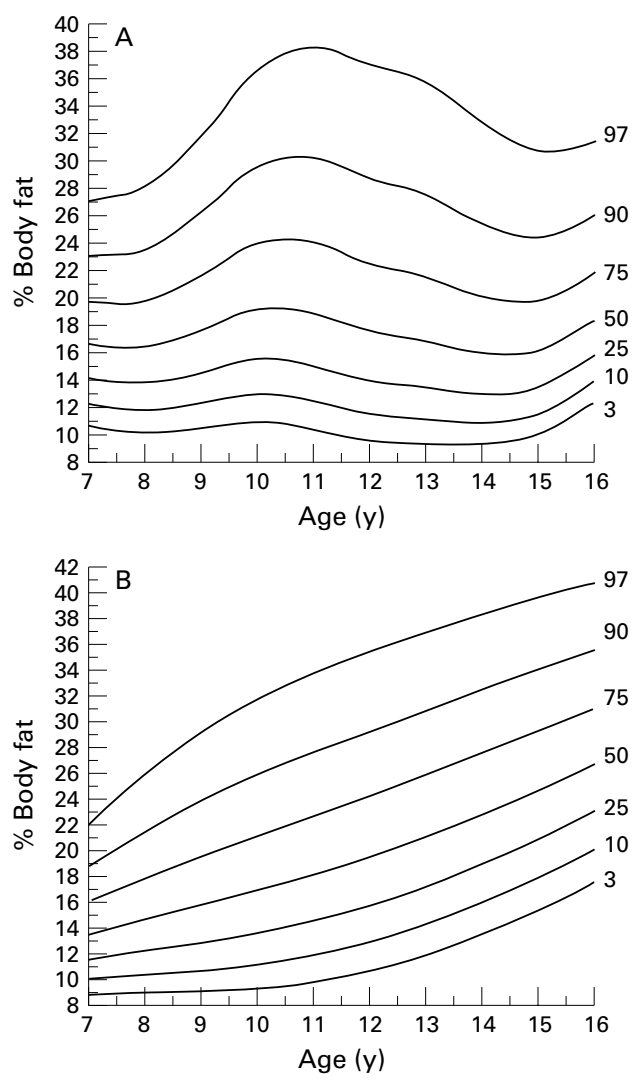

Figure 2 Percentage body fat in 1243 boys $(A)$ and 1139 girls (B).

\section{Discussion}

DXA is a well recognised standard method for determining body fat. ${ }^{6}$ We used DXA to validate the simpler leg to leg BIA method for measuring body fat. Our results suggest that leg to leg BIA measurements provide a valid estimate of body fat in Hong Kong children aged 7-16 years, albeit with some overestimation. Several studies in adults have compared estimated body composition from both conventional arm to leg BIA and newer leg to leg BIA devices with DXA, and found reasonable agreement. $^{8-10}$ Nunez and colleagues also reported good agreement between conventional arm to leg electrode BIA and the leg to leg device. ${ }^{9}$ Two previous studies have reported body composition determinations using arm to leg BIA in Hong Kong Chinese children and reached differing conclusions about the validity of this method. ${ }^{112}$ One study compared BIA with skinfold anthropometric predictions in 94 normal children aged 11-17 years; BIA was found to be a reliable and acceptably accurate method, which could be used on subjects for whom skinfold thickness is difficult to measure. ${ }^{11}$ The other study compared body composition measurement by magnetic resonance imaging, skinfold anthropometric prediction, and BIA in 19 obese and non-obese children aged $8-12$ years (11 subjects were aged 11 years). ${ }^{12}$ This study concluded that BIA had a relatively low correlation with total body fat and percentage of body fat and, unless combined with BMI in a prediction equation, its use alone in assessing obesity was not recommended. The latter study however was limited by a small sample size and used a conventional tetrapolar BIA device.

Gutin and colleagues ${ }^{7}$ reported that BIA underestimates body fat compared to DXA, whereas our results showed that BIA overestimated fat mass by a mean of $1.93 \mathrm{~kg}$. We also found much narrower limits of agreement. These differences might relate to different BIA devices and the in built prediction equations. It was not possible to obtain details of these prediction equations from the Tanita company, but presumably differences in these equations could result in measurement differences between different machines. We would therefore agree with the suggestion of Gutin et al that different methods should not be used interchangeably. ${ }^{7}$ For standardisation purposes it might also be necessary to use similar machines from a single manufacturer. It is known that BIA measurements may be affected by meals, physical activity, and other variables that change the subject's hydration state. Performance ot BIA three to five hours after a meal could lead to a significant underestimation of body fat mass. ${ }^{13}$ It is therefore suggested that ideally BIA should be measured in the morning, in a fasting state, and after emptying the bladder. ${ }^{814}$ For practical reasons we were unable to undertake measurements in the morning in a fasting state, but we did take the BIA measurements two hours postprandially. The timing of the BIA measurements in the study of Gutin et al is not documented. Differences between their results and ours could relate to differences in timing of measurements or to differences in BIA devices.

Ethnicity is another factor that may explain these differences. A study comparing the BIA index (height ${ }^{2} /$ resistance), total body water, height, weight, age, and gender of 125 white people and 89 African-Americans between 14 and 53 years old found that the prediction equations for total body water differ between the two racial groups. ${ }^{15}$ Another study assessing the association between ethnicity, body mass index, and bioelectrical impedance in subjects of 10 different ethnic origins also concluded that group specific references are needed when applying BIA. ${ }^{16}$

Using the proposed international BMI cut off value (BMI-25INT), 18\% of boys and $12 \%$ of girls in our study were classified as overweight. Using the same BMI-25INT cut off it has been shown that in the 1993 Hong Kong survey, only $11.7 \%$ of boys and $9.8 \%$ of girls were overweight, ${ }^{4}$ suggesting that during the past seven years the problem of overweight children in Hong Kong has increased; the effect is more noticeable in boys than in girls. We cannot, however, exclude the alternative explanation that the children recruited to this study were not representative of the whole paediatric population in Hong Kong. The male preponderance of overweight in Hong Kong is in contrast to a previous report that boys are generally leaner than girls at all ages. ${ }^{17}$ Reports from other Asian countries (Singapore and Taiwan) have shown that in these countries a greater percentage of males than females are overweight. ${ }^{18} 19$ 
Leg to leg BIA data from 2382 children aged 7-16 years were used to construct percentage body fat references curves and body fat cut off points, corresponding to BMI cut off values that would classify children as overweight or obese. There were notable differences in the shape of the body fat centile curves for boys and girls (fig 2). Body fat percentage in boys increased from age 8 , peaked at age 11 , and levelled off at age 14, possibly influenced by pubertal changes. The height of the peak was more pronounced for the higher centiles. In contrast the body fat centiles for the girls increased more steadily from $7-16$ years (fig 2 ). It is not clear to what extent the shape of the body fat centile curves for the boys is a reflection of the increased proportion of overweight boys in this sample compared to the 1993 Hong Kong study. The body fat percentage cut off values to define overweight (table 6) show similar differences between boys and girls. The cut off values for boys appear less consistent, varying upwards and downwards between $17.6 \%$ and $25.5 \%$, whereas those for girls show a more steady increase from $20.5 \%$ to $35.5 \%$ with increasing age. This gender specific pattern is similar to that previously reported by Ellis and colleagues for fat mass in boys and girls. ${ }^{20}$ This study compared reference models to measured fat mass; in boys this showed an upward bulge between the ages of 9 and 16 years. Whether this bulge represents a normal physiological pattern or whether it represents a tendency to overweight in boys in the study population is not clear. The increase of fat mass in girls in this study was similar to our data. In addition the study of Ellis et al showed that the measured fat mass was generally greater than that predicted from the reference models.

The relation between percentage body fat and BMI differs among different ethnic groups. ${ }^{21}$ This implies that if percentage body fat cut off values derived from BIA measurements for obesity are to be clinically useful for overweight, it would be desirable to collect more BIA and BMI data for boys and girls from different ethnic backgrounds and from a wider range of age groups with standardised BIA devices.

In conclusion, this study has validated a leg to leg BIA device against DXA and then presented BIA derived normative data on the percentage of body fat according to age and gender. Based on BMI standards, preliminary age and gender related cut off values values for percentage body fat were presented that would define overweight and obesity in this population. The leg to leg BIA method has potential for widespread use because of its simplicity and acceptable validity. The relatively good agreement between DXA and BIA measurement of body composition suggests that it is a useful method for cross sectional and longitudinal studies. However, some degree of overestimation of percentage body fat was noted and more information on possible variations in body fat measurements obtained from BIA machines from different manufacturers and used in different ethnic groups would be useful.

The work described in this paper was substantially supported by a grant from the Research Grants Council of the Hong Kong Special Administrative Region (Project No.CUHK 4060/00M). We thank Dr HK So and Miss Y Wong for their help in the collection of data.

1 Bland JM, Altman DG. Measuring agreement in method comparison studies. Stat Methods Med Res 1999;8;135-60.

Cole TJ, Green PJ. Smoothing reference centile curves: the LMS method and penalized likelihood. Stat Med 1992;11: 1305-19.

3 Leung SSF, Cole TJ, Tse LY, Lau JTF. Body mass index reference curves for Chinese children. Ann Hum Biol 1998; 25:169-74.

4 Cole TJ, Bellizzi MC, Flegal KM, Dietz WH. Establishing a standard definition for child overweight and obesity standard definition for child overweight and o
worldwide: international survey. BMF 2000;320:1-6.

5 International Association for the Study of Obesity, WHO. The Asia-Pacific perspective: redefining obesity and its treatment. Australia: Health Communications Australia Pty Ltd, 2000:18.

6 Ellis KJ, Shypailo RJ, Pratt JA, Pond WG. Accuracy of dualenergy x-ray absorptiometry for body-composition measurements in children. Am f Clin Nutr 1994;60:660-5.

7 Gutin B, Litaker M, Islam S, et al. Body-composition measurement in 9-11 y-old children by dual-energy x-ray absorptiometry, skinfold-thickness measurements, and bioimpedance analysis. Am f Clin Nutr 1996;63:287-92.

8 Stewart SP, Bramley PN, Heighton R, et al. Estimation of body composition from bioelectrical impedance of body egment comparison with dual-energy X-ray absorptiom etry. Br f Nutr 1993;69:645-55.

9 Nunez C, Gallagher D, Visser M, et al. Bioimpedance analysis: evaluation of leg-to-leg system based on pressure analysis: evaluation of leg-to-leg system based on pressure $524-31$

10 Jebb SA, Cole TJ, Doman D, et al. Evaluation of the novel Tanita body-fat analyser to measure body composition by comparison with a four-compartment model. Br f Nutr 2000;83:115-22.

11 Eston RG, Cruz A, Fu F, Fung LM. Fat-free mass estimation by bioelectrical impedance and anthropometric techniques in Chinese children. F Sports Sci 1993;11: $241-7$

12 Chan YL, Leung SSF, Lam WWM, et al. Body fat estimation in children by magnetic resonance imaging, bioelectrical impedance,skinfold and body mass index: a pilot study. $\mathcal{F}$ Paediatr Child Health 1998;34:22-8.

13 Gallagher MR, Walker KZ, O'Dea K. The influence of a breakfast meal on the assessment of body composition using bioelectrical impedance. Eur f Clin Nutr 1998;52: using

14 Deurenberg P, Weststrate JA, Paymans I, van der Kooy K. Factors affecting bioelectrical impedance measurements in Factors affecting bioelectrical impedance meas
humans. Eur f Clin Nutr 1988;42:1017-22.

15 Schoeller DA, Luke A. Bioelectrical impedance analysis prediction equations differ between African Americans and Caucasians, but it is not clear why. Ann N Y Acad Sci 2000; 904:225-6.

16 Ward LC, Heitmann BL, Craig P, et al. Association between thnicity, body mass index, and bioelectrical impedance. Ann N Y Acad Sci 2000;904:199-202.

17 Ogle GD, Allen JR, Humphries IRJ, et al. Body-composition assessment by dual-energy x-ray absorptiometry in subjects aged 4-26 years. Am f Clin Nutr 1995;61:746-53.

18 Rajan U. Obesity among Singapore students. Int 7 Obes 1994;18(suppl 2):27.

19 Peng CJ, Chang CM, Kuo SE, et al. Analysis of anthropometric growth trends and prevalence of abnormal body status in Taiwan elementary-school children. Taiwan Erh Ko I Hsueh Hui Tsa Chih 1999;40:406-13.

20 Ellis KJ, Shypailo RJ, Abrams SA, Wong WW. The reference child and adolescent models of body composition. A contemporary comparison. Ann N Y Ácad Sci 2000;904: 374-82.

21 Deurenberg P, Yap M, van Staveren WA. Body mass index and percentage body fat: a meta analysis among different ethnic groups. Int $\mathcal{F}$ Obes Relat Metab Disord 1998;22:116471 . 